\title{
Multiple Model Adaptive Control of neuromuscular blockade: Design guidelines and clinical cases
}

\author{
Teresa Mendonça, Hugo Magalhães, João M. Lemos, Paula Rocha and Simão Esteves
}

\begin{abstract}
The high level of uncertainty of the dynamic response of patients subject to anaesthesia motivates the use of adaptive control methods. This paper proposes an approach based on Switched Multiple Model Adaptive Control (SMMAC) to tackle this problem in what concerns the control of the neuromuscular blockade level. It is shown how to design the different elements of the SMMAC controller, enhancing the importance of the observer polynomial, that is shown to be instrumental to stabilize the loop. Clinical results using atracurium as blocking agent are reported, thereby illustrating the application of the proposed approach in actual clinical practice.
\end{abstract}

Index Terms - Anaesthesia, Neuromuscular Blockade, Computer Control, Adaptive Control, Biomedicine, control applications.

\section{INTRODUCTION}

Feedback control for drug dosing in clinical pharmacology is receiving increasing attention [1], [2]. Besides circumventing the tedious, imprecise and sometimes low performance procedures associated to open-loop control, feedback control can also significantly advance the medical understanding of the effects of pharmacology agents and anesthetics and provide progress in drug delivery systems. Significant examples [1] are provided by the closed-loop control of the cardiovascular function and automated anaesthesia. During surgical procedures patients are usually under general anaesthesia, defined as the lack of response and recall to noxious stimuli, reflected in loss of conscience, pain insensitivity and muscle paralysis. Several approaches to the neuromuscular blockade control problem as well as an introduction to this issue can be seen in [3], [6], [4] [7] and [5]. Muscle relaxant drugs are frequently given during surgical operations. The non-depolarizing types of muscle relaxant act by blocking the neuromuscular transmission (NMT), thereby producing muscle paralysis. The level of muscle relaxation is measured from an evoked EMG at the hand by electrical stimulation of the adductor policies muscle to supra-maximal train-of-four stimulation of the ulnar nerve. In a clinical environment the measurement of the neuromuscular blockade level corresponds to the first single response $\left(T_{1} \%\right)$ calibrated by a reference twitch,

This paper was written in relation with the NISIS EC network. T. Mendonça and H. Magalhães are with the DMA, FCUP, Rua do Campo Alegre, 687, 4169-007 Porto, Portugal. \{tmendo, hfmagalh \} @ f C.up.pt

J.M. Lemos is with the INESC-ID/IST, Rua Alves Redol, 9, 1000-029 Lisboa, Portugal. jlml@inesc.pt

P. Rocha is with the DM, UA, Campo de Santiago, 3810-193 Aveiro, Portugal. procha@mat.ua.pt

Simão Esteves is with the Anestesiology Department, Hospital Geral de Santo António, Largo Prof. Abel Salazar, 4050 Porto, Portugal. obtained by defining a supra-maximal stimulation current. This measuring process is prone to the occurrence of outliers, a problem dealt with in [8]. The control of the neuromuscular blockade provides a good illustration of the main features and inherent constraints associated with the control of physiological variables. It is characterized by a very high degree of uncertainty in the dynamics of the system due both to interpatient variability as well as time variations. This suggests that multiple-models based control techniques would provide a suitable solution [9]-[11]. Such a scheme (incorporating several modifications in order to accommodate the specific characteristics of the problem) has been developed in [12], using multiple controllers constructed from a bank of models which replicate the observed variability of the dynamic responses to the muscle relaxant [13]. In [14] restriction techniques (controller localization) relying on a robustness condition have been proposed. These techniques yield good results but suffer from the major drawback of assuming a strong assumption on the plant dynamics.

The main contribution of this paper consists in providing guidelines for designing the Switched Multiple Model Adaptive Control algorithm for neuromuscular blockade using atracurium, and to validate them with actual clinical cases. A special emphasis is placed in the selection of the observer dynamics, for which guidelines for the selection of the characteristic polynomial are provided.

The paper is organized as follows: after the introduction which motivates and formulates in general terms the problem to be solved, the model for neuromuscular blockade is described in section 2 . The switching multiple model control algorithm used is described in section 3. Section 4 describes results, both simulations and clinical trials. Finally, section 5 draws conclusions.

\section{NeUROMUSCULAR BLOCKADE MODEL}

In order to design the bank of local controllers upon which the switched multiple model algorithm relies. Furthermore, this model reveals the structure of the system to control, thereby providing insight on design options.

The dynamic response of the neuromuscular blockade for atracurium may be modelled as shown in Figure 1 [7], [13]. A linear pharmacokinetic model (block 1 of Figure 1), described by the following linear system of state equations

$$
\left\{\begin{array}{l}
\dot{x}_{1}(t)=-\lambda_{1} x_{1}(t)+a_{1} u(t) \\
\dot{x}_{2}(t)=-\lambda_{2} x_{2}(t)+a_{2} u(t) \\
c_{p}(t)=\sum_{i=1}^{2} x_{i}(t),
\end{array}\right.
$$




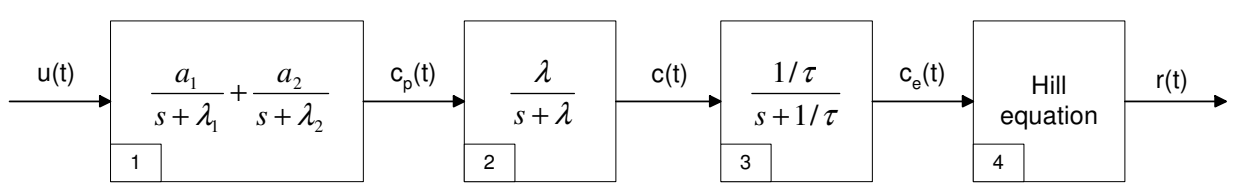

Fig. 1. Block diagram of the neuromuscular blockade model.

relates the drug infusion rate $u(t)\left[\mu g \mathrm{~kg}^{-1} \mathrm{~min}^{-1}\right]$ with the plasma concentration $c_{p}(t)\left[\mu g m l^{-1}\right]$, where $x_{i}(i=$ $1,2)$ are state variables (implicitly defined by (1)) and $a_{i}$ $\left[\mathrm{kg} \mathrm{ml}^{-1}\right], \lambda_{i}\left[\mathrm{~min}^{-1}\right](i=1,2)$ are patient dependent parameters. The physiological basis of the model described by equation (1) consists of assuming two plasma compartments (central and peripheric) both communicating with each other. A linear second order model (blocks 2 and 3 of Figure 1), described by the cascade of two first order systems, written as

$$
\dot{c}(t)=-\lambda c(t)+\lambda c_{p}(t)
$$

and

$$
\dot{c}_{e}(t)=-1 / \tau c_{e}(t)+1 / \tau c(t),
$$

is assumed to relate $c_{p}(t)$ with the concentration in the effect compartment, $c_{e}(t)\left[\mu g \mathrm{ml}^{-1}\right]$. Here, $c(t)$ is an intermediate variable and $\lambda\left[\mathrm{min}^{-1}\right], \tau[\mathrm{min}]$ are patient dependent parameters. It is remarked that standard models developed for atracurium [?], [15] do not consider the block 3. As shown in [13], the inclusion of the extra delay associated to $\tau$ allows a better replication of the observed experimental responses. Finally the pharmacodynamic effect, that relates $c_{e}(t)$ to the induced pharmacodynamic response, $r(t)[\%]$, may be modelled by the Hill equation [15](block 4 of Figure $1)$

$$
r(t)=100 C_{50}^{\gamma} /\left(C_{50}^{\gamma}+c_{e}^{\gamma}(t)\right),
$$

where the parameters $C_{50}\left[\mu \mathrm{gml}^{-1}\right]$ and $\gamma$ (adimensional) are also patient-dependent. The variable $r(t)$, normalized between 0 and 100, measures the level of the neuromuscular blockade, 0 corresponding to full paralysis and 100 to full muscular activity.

\section{SWITCHING CONTROL}

In recent years adaptive control approaches based on supervisory switching control have been proposed to deal with systems presenting high level of uncertainty. This section describes the basic structure of switching control and presents two different solutions to overcome robustness issues related to the implementation of such a control scheme. It is assumed that a discretization procedure has taken place, and hence from now on the variable $t$ represents discrete time instants.

\section{A. Basic structure}

The basic structure of a supervisor based switched multiple model controller is seen in Figure 2, as described in [9], [10], [14]. In Figure 2, $y$ denotes the sensor measure, ref denotes the reference, $e_{c}$ the control error and $P$ the plant to be controlled. A bank of controllers $C_{j}, j=1, \ldots, N$ is designed to match the plant models $M_{j}$. This set of models is assumed to "cover" all the possibilities of the actual plant $P$. In order to select at each time which controller best matches $P$, the principle according to which the best model performance implies the best controller performance is applied. One possibility for evaluating the model performance is to compare the output $y_{j}$ of each model $M_{j}$ with the process output $y$. Another possibility is to construct estimators $E_{j}$ based on each of the models $M_{j}$, see subsection III-C.

In either case an error $e_{j}$ is produced, which is measured through a performance index (PI) $\pi_{j}, j=1, \ldots, N$, computed by low pass filtering according to

$$
\pi_{j}(t+1)=\lambda_{\pi} \pi_{j}(t)+\left(1-\lambda_{\pi}\right) e_{j}(t),
$$

where $t$ is discrete time and $\lambda_{\pi}$ is a parameter to adjust. The switching logic block SL selects the index $\sigma$ of the controller to apply to the plant. This selection is given by the value of $j$ corresponding to the least value of $\pi_{j}$, but ensuring that, once a controller is applied to the plant, it remains so for a minimum amount of time $\tau_{D}$. This is the so called "dwell time" condition, which prevents high frequency commutation among controllers and prevents instabilities that could occur due to too fast switching [9]. An integrator common to all blocks ensures bumpless transfer between different controllers [9] (discrete control transfer function $C_{j}(z)=$ $\bar{C}_{j}(z) \frac{z \Delta t}{z-1}, \quad \Delta t$ sampling time). Outlier removal is performed with a Bayesian filter, according to the techniques described in [8].

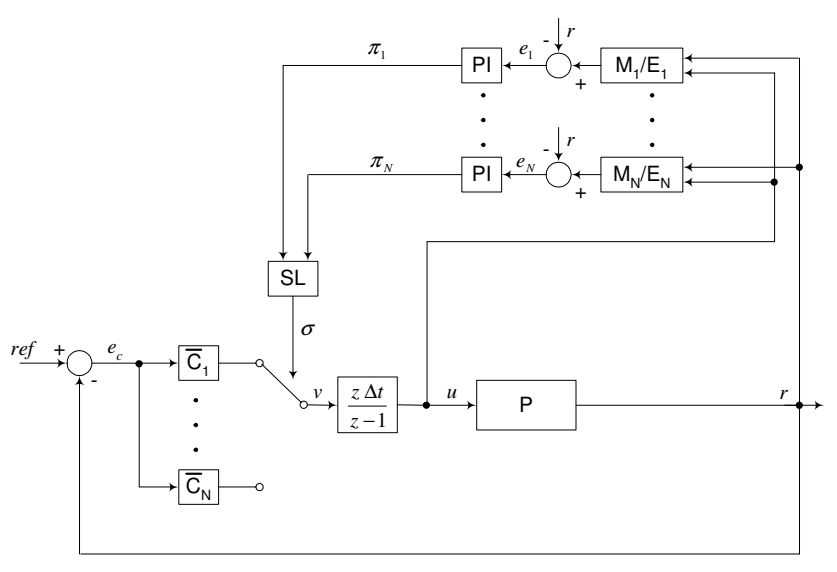

Fig. 2. Switched multiple model control strategy. 


\section{B. Local controller design}

Each controller $C_{j}$ in the bank of local controllers has a PID structure with the integrator separated, parameterized as

$$
\begin{aligned}
& v(t)=k_{p}\left(\frac{1}{T_{i}}+\frac{z-1}{z \Delta t}+T_{d}\left(\frac{z-1}{z \Delta t}\right)^{2}\right) e_{c}(t), \\
& u(t)=\frac{z \Delta t}{z-1} v(t) .
\end{aligned}
$$

The gains $k_{p}, T_{i}$ and $T_{d}$ are designed according to a dominant-pole placement rule [16] by applying the expressions:

$$
\begin{aligned}
n & =b_{1} w_{1}-b_{2} w_{2}+\zeta a_{2} w_{2}, \\
a & =\frac{a_{2}-a_{1}+\zeta b_{2}}{\alpha n}, \\
b & =\frac{b_{2} w_{1}-b_{1} w_{2}-\zeta a_{2} w_{1}}{\alpha w_{1} w_{2} n}, \\
T_{i} & =-\frac{a}{2} \sqrt{\frac{a^{2}}{4}-b}, \\
c & =-\zeta a_{2}+b_{2}-b_{1}, \\
d & =\left(a_{2}+\zeta b_{2}\right)\left(w_{2} \alpha T_{i}-\frac{1}{w_{2} T_{i}}\right), \\
e & =-a_{1}\left(w_{1} \alpha T_{i}-\frac{1}{w_{1} T_{i}}\right), \\
k_{p} & =\frac{\zeta}{c+d+e}, \\
T_{d} & =\alpha T_{i},
\end{aligned}
$$

where $G\left(j w_{i}\right)=a_{i}+j b_{i}, i=1,2$ are two points of the plant Nyquist curve, $\zeta$ is the damping ratio and $\alpha$ is $a$ priori fixed. Typical points of the Nyquist curve are those which correspond to the plant phase frequencies $-180^{\circ}$ and a value close, but inferior to it, and a common value for $\alpha$ is $\alpha=4$. The above method for designing PIDs proves to be an adequate one, even for non-minimum phase plants and provides a step response with little overshoot.

\section{Observer Dynamics}

As done in [9], the estimate $\hat{y}^{j}(t)$ of $y(t)$ is made by including an observer polynomial.

Let each model $M_{j}$ be represented by the ARX model

$$
A_{j}\left(q^{-1}\right) y_{j}(t)=B_{j}\left(q^{-1}\right) u(t)+\bar{e}_{j}(t),
$$

in which

$$
\begin{aligned}
& A_{j}\left(q^{-1}\right)=1+\sum_{i=1}^{n_{a}} a_{j, i} q^{-i}, \\
& B_{j}\left(q^{-1}\right)=\sum_{i=1}^{n_{b}} b_{j, i} q^{-i}
\end{aligned}
$$

are polynomials in the unit delay operator $q^{-1}$, with $A_{j}$ monic and $B_{j}$ of fixed degrees $n_{a}$ and $n_{b}$ respectively, for all $j=1, \ldots, N$. In order to associate to (11) a state space model, define $\left(n_{b}>1\right)$

$$
\begin{aligned}
& s(t)= \\
& {\left[y_{j}(t) \ldots y_{j}\left(t-n_{a}+1\right) u(t-1) \ldots u\left(t-n_{b}+1\right)\right]^{T} .}
\end{aligned}
$$

For $n_{b}=1, s(t)=\left[y_{j}(t) \ldots y_{j}\left(t-n_{a}+1\right)\right]$. In the case of an ARX model, i.e. when the disturbance acting on (11) is white noise, $s(t)$ is a (nonminimal) state associated to (11), whose evolution is described by the state-space model

$$
\begin{aligned}
s(t+1) & =\Phi_{j} s(t)+\Gamma_{j} u(t)+G \bar{e}(t+1) \\
y_{j}(t) & =H s(t)
\end{aligned}
$$

in which

$$
\begin{gathered}
\Phi_{j}= \\
{\left[\begin{array}{cccccc}
-a_{j, 1} & \ldots & -a_{j, n_{a}} & b_{j, 2} & \ldots & b_{j, n_{b}} \\
0 & I_{n_{a}-1} & 0_{n_{a}-1 \times 1} & 0 & 0_{n_{a}-1 \times n_{b}-2} & 0_{n_{a}-1 \times 1} \\
0 & \cdots & 0 & 0 & \ldots & 0 \\
& 0 & 0_{n_{b}-2 \times 1} & & I_{n_{b}-2} & 0_{n_{b}-2 \times 1}
\end{array}\right]} \\
\Gamma_{j}=\left[\begin{array}{lllll}
b_{j, 1} & 0_{1 \times n_{a}-1} & 1 & 0_{1 \times n_{b}-2}
\end{array}\right]^{T},
\end{gathered}
$$

with the " 1 " in $\Gamma_{j}$ in the $n_{a}+1$ th position $\left(n_{b}>1\right)$ and $G=$ $\left[\begin{array}{ll}1 & 0_{1 \times n_{a}+n_{b}-2}\end{array}\right]^{T}, H=\left[\begin{array}{ll}1 & 0_{1 \times n_{a}+n_{b}-2}\end{array}\right]$. The pair $\left(\Phi_{j}, \Gamma_{j}\right)$ is reachable and $\left(H, \Phi_{j}\right)$ is reconstructible (the state can be obtained from past data) but not observable (the state cannot be recovered from future data). The advantage of using this type of state consists in the fact that it readily provides a shared state realization, i.e., a state-space realization in which the state is common to all models $M_{j} / E_{j}$ in Figure 2. Consider now the problem of designing a state observer to (15) or equivalently, a prediction to (11). For that sake add $A_{o} y_{j}(t)-A_{j} y_{j}(t)$ to both sides of (11) to conclude that the model may be represented by

$$
y_{j}(t)=\left(A_{o}-A_{j}\right) \frac{1}{A_{o}} y_{j}(t)+\frac{B_{j}}{A_{o}} u(t)+\frac{1}{A_{o}} \bar{e}_{j}(t),
$$

where $A_{o}\left(q^{-1}\right)=1+\sum_{i=1}^{n_{a}} a_{o, i} q^{-i}$ is a stable monic polynomial with the same degree as the $A_{j}$ 's, which will hereafter be referred as the "observer polynomial". Define an estimator $E_{j}$ for the plant by

$$
\hat{y}_{j}(t)=\left(A_{o}-A_{j}\right) \frac{1}{A_{o}} y(t)+\frac{B_{j}}{A_{o}} u(t) .
$$

The idea behind this is that if the process model would coincide with $M_{j}, y$ would coincide with $y_{j}$. Therefore

$$
y(t)-\hat{y}_{j}(t)=y_{j}(t)-\hat{y}_{j}(t)=\frac{1}{A_{o}} \bar{e}_{j}(t)
$$

and the estimate $\hat{y}_{j}$ would be asymptotically accurate provided that $A_{o}$ is chosen to be Hurwitz. Indeed, the tracking error is a low pass filtering of the disturbances. If a deadbeat ("fast") observer is selected (all the roots of $A_{o}$ at the origin) no filtering occurs. As confirmed by the experiments below, a much better choice consists in making the observer dynamics much slower, by selecting the roots of $A_{o}$ in the real segment between 0 and 1, but away from 0 .

\section{State space interpretation}

The estimator (19) can be interpreted in terms of a state observer as follows. Define

$$
\begin{aligned}
\eta(t) & =\frac{1}{A_{o}\left(q^{-1}\right)} y(t), \\
\nu(t) & =\frac{1}{A_{o}\left(q^{-1}\right)} u(t) .
\end{aligned}
$$


With the state defined by

$$
x_{\eta}(t)=\left[\begin{array}{llll}
\eta(t) & \eta(t-1) & \ldots & \eta\left(t-n_{a}+1\right)
\end{array}\right]^{T},
$$

the state-space realization of (21) is seen to be

$$
x_{\eta}(t)=\bar{\Phi}_{o} x_{\eta}(t-1)+\bar{\Gamma}_{o} y(t)
$$

where

$$
\begin{gathered}
\bar{\Phi}_{o}=\left[\begin{array}{cccc}
-a_{o, 1} & -a_{o, 2} & \cdots & -a_{o, n_{a}} \\
& I_{n_{a}-1} & & 0_{n_{a}-1 \times 1}
\end{array}\right], \\
\bar{\Gamma}_{o}=\left[\begin{array}{ll}
1 & 0_{1 \times n_{a}-1}
\end{array}\right]^{T} .
\end{gathered}
$$

For (22), a similar reasoning holds. The state defined by

$$
x_{\nu}(t)=\left[\begin{array}{llll}
\nu(t) & \nu(t-1) & \ldots & \nu\left(t-n_{a}+1\right)
\end{array}\right]^{T}
$$

yields

$$
x_{\nu}(t)=\bar{\Phi}_{o} x_{\nu}(t-1)+\bar{\Gamma}_{o} u(t) .
$$

In turn, defining the state of the reconstructor as

$$
s_{f}(t)=\left[\begin{array}{ll}
x_{\eta}(t)^{T} & x_{\nu}(t)^{T}
\end{array}\right]^{T},
$$

equation (24) together with (28) yield

$$
s_{f}(t)=\Phi_{o} s_{f}(t-1)+\Gamma_{o y} y(t)+\Gamma_{o u} u(t),
$$

in which

$$
\begin{gathered}
\Phi_{o}=\left[\begin{array}{cc}
\bar{\Phi}_{o} & 0_{n_{a} \times n_{a}} \\
0_{n_{a} \times n_{a}} & \bar{\Phi}_{o}
\end{array}\right], \\
\Gamma_{o y}=\left[\begin{array}{ll}
\bar{\Gamma}_{o}^{T} & 0_{1 \times n_{a}}
\end{array}\right]^{T}, \\
\Gamma_{\text {ou }}=\left[\begin{array}{ll}
0_{1 \times n_{a}} & \bar{\Gamma}_{o}^{T}
\end{array}\right]^{T} .
\end{gathered}
$$

From (19)

$$
\hat{y}_{j}(t)=H_{j, o} s_{f}(t-1)
$$

with $H_{j, o}$ given by

$$
H_{j, o}=\left[\begin{array}{lll}
a_{o, 1}-a_{j, 1} \ldots a_{o, n_{a}}-a_{j, n_{a}} & b_{j, 1} \ldots b_{j, n_{b}} & 0_{1 \times n_{a}-n_{b}}
\end{array}\right]
$$

The vector $s_{f}(t)$ corresponds to a filtering of $s(t)$ by the observer dynamics. This dynamics may be chosen in order to provide the controller with robustness properties with respect to un-modelled plant dynamics. A possibility consists in choosing a dead-beat observer, in which case $a_{o, i}=0$ for $i=1, \ldots, n$ and $s(t)$ is exactly recovered after $n$ steps. However, as already mentioned, the dead-beat observer is an inconvenient choice in the case of neuromuscular blockade where the possible dynamics are such that this may lead to a wrong selection of the best local controller.

\section{RESUlTS}

To achieve a high level of neuromuscular blockade in a short time, a bolus of atracurium is always administered in the beginning of a surgery. After the administration of the bolus, the level of the muscular blockade increases very quickly (the variable $r$, that measures muscular activity decreases), and full muscle paralysis is induced in a few minutes. Following that initial period, the control objective is to follow a specific reference profile with a final target value $r e f \equiv r e f_{0}$. The value of the reference profile is initially fixed at a low level (typically $2.5 \%$ ) during the first 30 minutes. It is then gradually increased to the final value (typically 10\%). A bank of $\mathrm{N}=100$ non-linear dynamic models $M_{j}, j=1, \ldots, N$ with the same structure as described in section II was generated using the probabilistic model for atracurium [13]. Furthermore, for each $M_{j}$, a controller $C_{j}$ was tuned using the dominant-pole placement rule described in subsection III-B. This provides a controller bank which covers a wide range of behaviors.

A number of simulations are shown hereafter which illustrate the results obtained with switching control.

\section{A. Base line examples}

In this set of simulations, a base line is established and the observer polynomial is selected with all the roots at the origin (no filtering). Figure 3 illustrates the results for $P=M_{69}$, where the pair $\left(M_{69}, C_{69}\right)$ has been removed from the model-controller bank. As seen in Figure 3 the resulting behavior is poor.
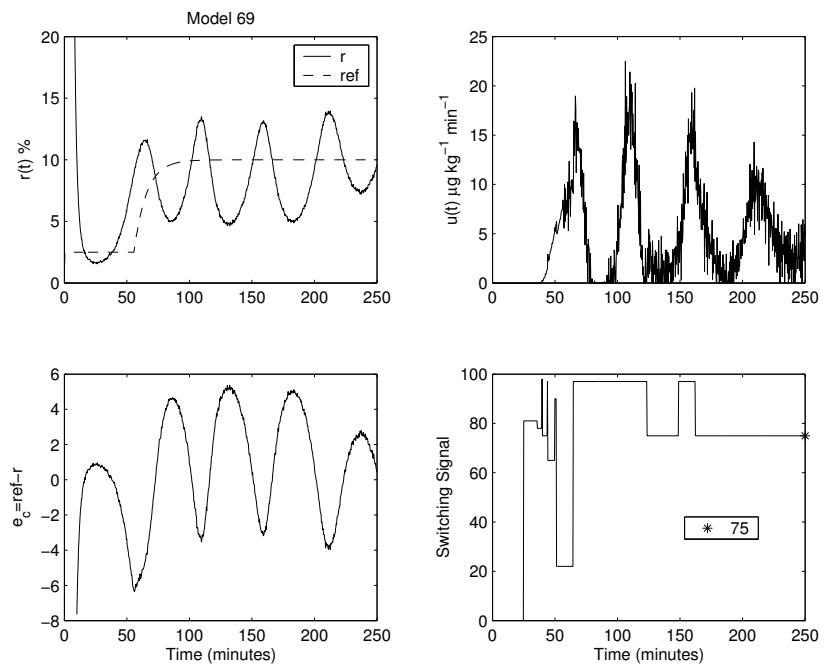

Fig. 3. Results obtained for $P=M_{69}$ with all the roots of the observer polynomial selected at the origin (no filtering).

\section{B. Observer dynamics effect}

If the roots of the observer polynomial are chosen to be stable and real but not at the origin, the performance clearly improves as expected. Two examples may be seen in Figures 4 and 5 with roots of $A_{o}$ at $\{0.85,0.85,0.85,0.85\}$ and $\{0.92,0.92,0.92,0.92\}$ respectively. 

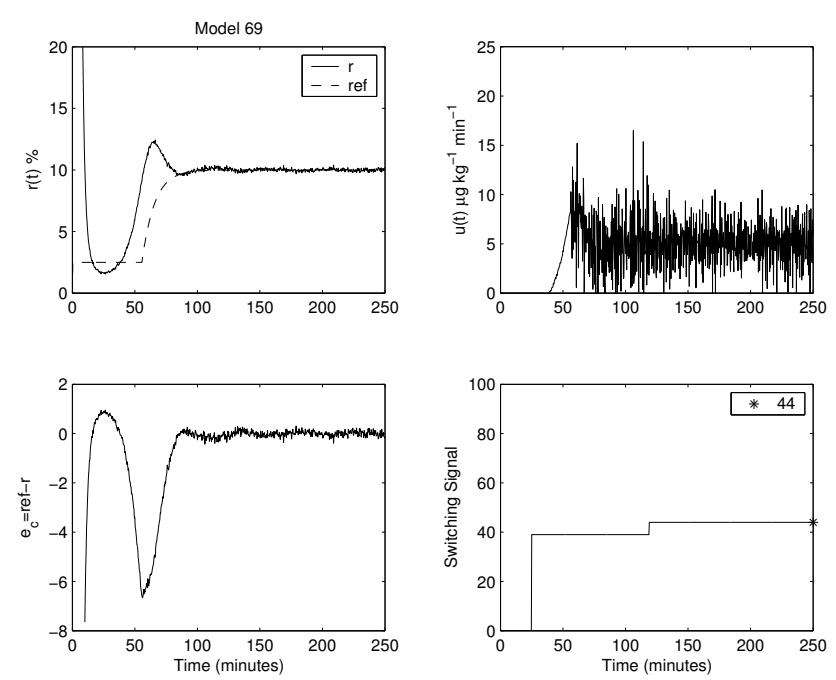

Fig. 4. Results obtained for $P=M_{69}$ with observer dynamics where the roots of $A_{o}$ are $\{0.85,0.85,0.85,0.85\}$.
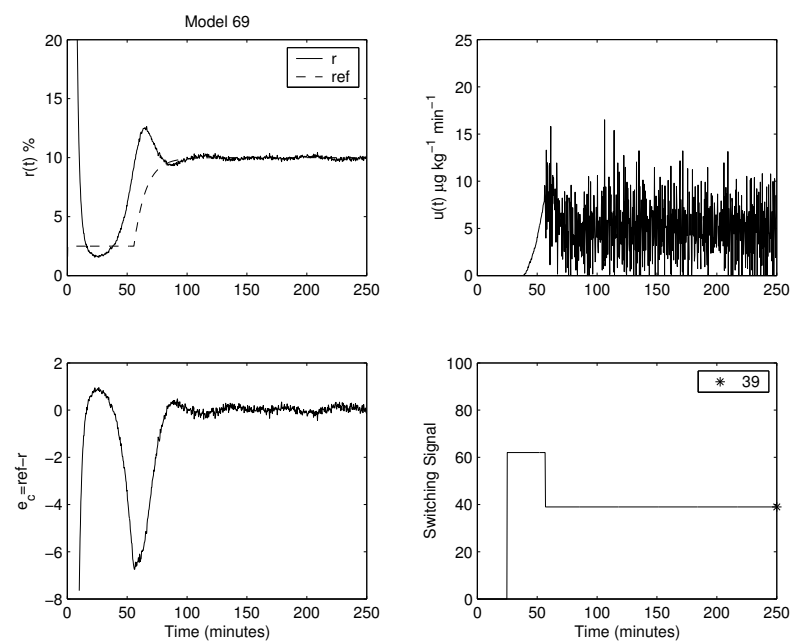

Fig. 5. Results obtained for $P=M_{69}$ with observer dynamics where the roots of $A_{o}$ are $\{0.92,0.92,0.92,0.92\}$.

An extensive simulation study using each model of the bank for mimicking the real plant $P$ was conducted. This study, based on the mean square tracking error (MSE) and initial overshoot value, proved the superior performance of the controller designed when $A_{o}$ roots $r_{i}^{o} \in[0.65,0.95], i=$ $1, \ldots, n_{a}=4$ (order of the neuromuscular blockade model). All roots of $A_{o}$ were made equal. Figure 6 shows the MSE as a function of the roots $r_{i}^{o}$ of the observer polynomial $A_{o}$ for the worst case observed, i.e., for $P=M_{69}$.

\section{Clinical results}

The clinical test of the above algorithm was performed as part of a study to evaluate and compare the performance of different control strategies, including SMMAC, approved by the Ethics Committee of Hospital Geral de Santo António (Porto, Portugal). In this framework, patients with health features levels I to IV according to the American Society

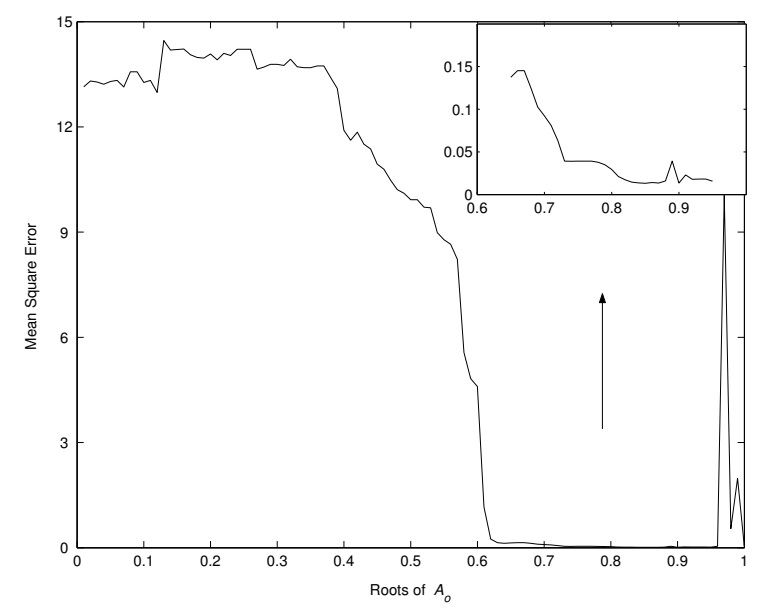

Fig. 6. Mean square error (MSE) for $P=M_{69}$ as a function of the roots $r_{i}^{o}$ of the observer polynomial $A_{o}$. The upper right corner shows a zoom of the MSE for $0.65 \leq r_{i}^{o} \leq 0.95$.

of Anesthesiology (ASA) have undergone elective surgery with automatic control of neuromuscular blockade.

Anesthesia was induced with intravenous fentanyl and propofol. After calibration of the NMT module [17] of the Datex AS/3 Anaesthesia Monitor, a $500 \mu \mathrm{g} \mathrm{kg}^{-1} \mathrm{~min}^{-1}$ bolus of atracurium was administered. Anesthesia was maintained with AIR or $\mathrm{N}_{2} \mathrm{O} / \mathrm{O}_{2}$, propofol infusion or sevoflurane and fentanyl as needed. Atracurium $(1 \mathrm{mg} / \mathrm{ml})$ was delivered by a computer controlled syringe driver (B|Braun Perfusor compact S) [18]. The controller is implemented in a portable battery operated computer that receives the muscle relaxation level signal via the RS $232 \mathrm{C}$ port, from the Datex AS/3 and updates the diffusion rate delivered by the pump with a sampling rate of $20 \mathrm{~s}$. The control algorithms were programmed in $C$-code, automatically generated from MATLAB code.

Figure 7 provides a view of a patient in the operating room with the set-up for automatic neuromuscular blockade control (NMB). The NMB sensor is seen on the left hand of the patient. The syringe is seen mounted on the computer controller drive (left of the picture, of green color) as well as the portable computer (on the right).

Figures 8 and 9 show clinical results with observer dynamics where the roots of $A_{o}$ are $\{0.85,0.85,0.85,0.85\}$ and $\{0.92,0.92,0.92,0.92\}$ respectively. The last situation (with the observer roots at 0,92 ) has clearly a superior performance, both in tracking the reference and in reducing the fluctuations of the manipulated variable. Again this illustrates the advantage of a slower observer.

\section{Conclusions}

The control of neuromuscular blockade during anaesthesia, through the continuous infusion of a muscle relaxant, is characterized by a very high degree of uncertainty in the dynamics of the system, due both to inter-patient variability as well as time variations. In order to deal with these features adaptation methods using multiple model based switching control was used. The selection of the observer dynamics was 


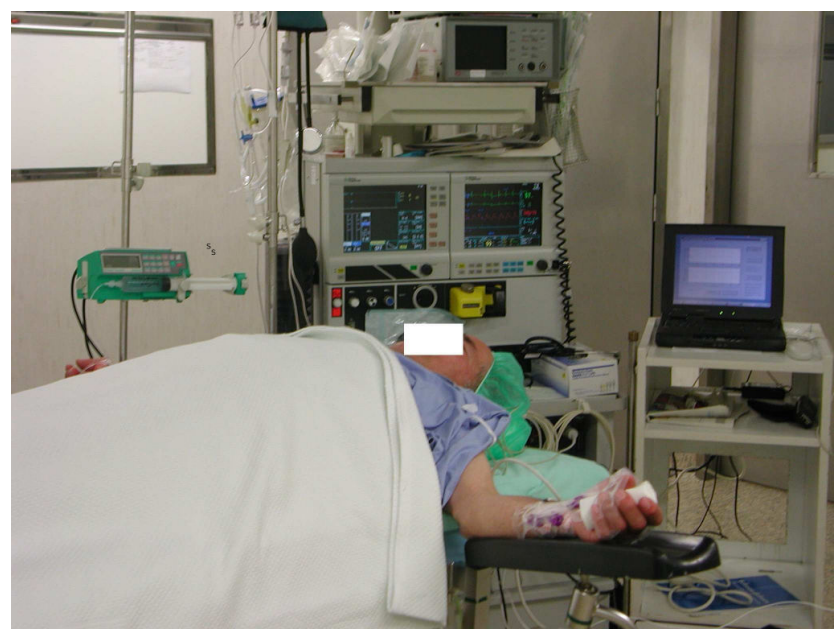

Fig. 7. A view of a patient in the operating room with the set-up for automatic neuromuscular blockade control.
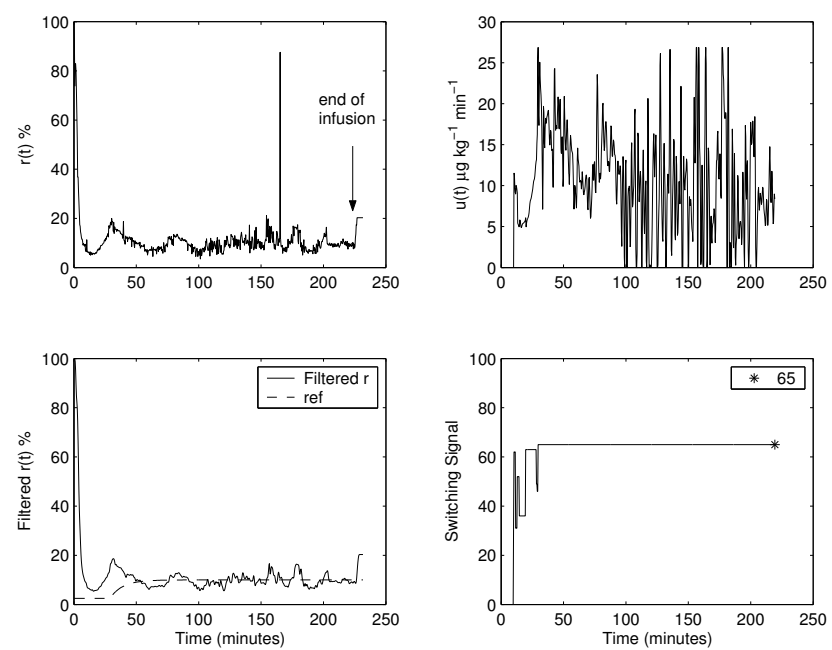

Fig. 8. Clinical results obtained with observer dynamics where the roots of $A_{o}$ are $\{0.85,0.85,0.85,0.85\}$.

shown to be a crucial issue for achieving good performance and even stabilizing the loop. As expected from theory, fast observers yield oscillating behaviors and may even lead to wrong a selection of the local controller. Another important aspect is the way local controllers are designed which reflects in the overall performance of SMMAC. In this paper, the local controllers are PIDs tuned according to a dominant pole placement rule. The clinical results confirmed the simulations and present a good performance. The algorithm was able to stabilize the patient dynamics without imposing any a priori restrictions on the bank of models/controllers.

\section{REFERENCES}

[1] J. Bailey and W. Haddad, Drug dosing in clinical pharmacology: paradigms, benefits, and challenges, IEEE Control Syst. Mag., vol. 25(2), 2005, pp 35-51.

[2] Araki, M. and E. Furutani (2005). Computer control of physiological states of patients under and after surgical operation. Annual Reviews in Control, 29:229-236.
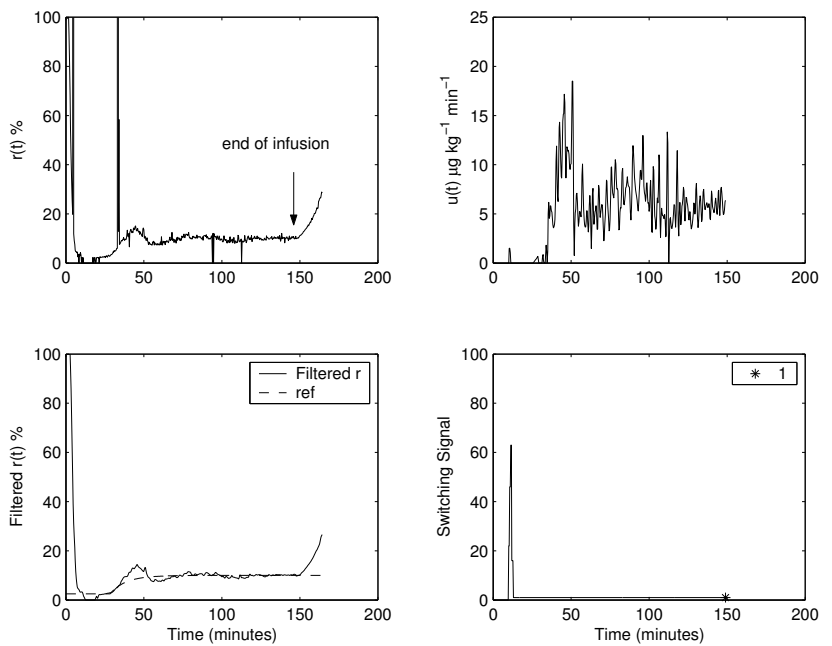

Fig. 9. Clinical results obtained with observer dynamics where the roots of $A_{o}$ are $\{0.92,0.92,0.92,0.92\}$.

[3] Jaklitsch, R. and D. Westenskov (1987). A model based self adjusting two-phase controller for vecuronium-induced muscle relaxation during anesthesia. IEEE Trans. Biom. Eng., BME-34:583-594.

[4] Kansanaho, M. and K. Olkkola (1996). Performance assessment of an adaptive model-based feedback controller: Comparison between atracurium, mivacurium, rocuronium and vecvuronium. J. Clin. Monitor., Comput., 13(4):217-224.

[5] Mason, D.; J. Ross, N. Edwards, D. Linkens and C. Reilly (1999). Self-learning fuzzy control with temporal knowledge for atracuriuminduced neuromuscular block during surghery. Comput Biomed. Res., 32(3):187-197.

[6] D. Linkens, Intelligent Control in Biomedicine, Taylor and Francis, 1994.

[7] T. Mendonça and P. Lago, PID control strategies for the automatic control of neuromuscular blockade, Control Eng. Pract., vol. 6(10), 1998, pp 1225-1231.

[8] J. M. Lemos and H. Magalhães and T. Mendonça and R. Dionísio, Control of neuromuscular blockade in the presence of sensor faults, IEEE Trans. Biomed. Eng., vol. 52(11), 2005, pp 1902-1911.

[9] S. Morse, Supervisory control of families of linear set-point controllers - Part 1: Exact matching, IEEE Trans. Automat. Contr., vol. 41(10), 1996, pp 1413-1431.

[10] K. Narendra and J. Balakrishnan, Adaptive control using multiple models, IEEE Trans. Automat. Contr, vol. 42(2), 1997, pp 171-187.

[11] Mosca, E. and T. Agnoloni (2001). Inference of candidate loop performance and data filtering for switching supoervisory control. Automatica, 37:527-534.

[12] A. Neves and T. Mendonça and P. Rocha, "Tracking by switching control: a case study", in Proc. $4^{\text {th }}$ Portuguese Conference on Automatic Control (CONTROLO'O0), Guimarães, Portugal, 2000, pp. 61-65.

[13] P. Lago and T. Mendonça and L. Gonçalves, "On-line autocalibration of a PID controller of neuromuscular blockade", in Proc. of the 1998 IEEE International Conference on Control Applications, Trieste, Italy, 1998, pp. 363-367.

[14] T. Mendonça and P. Lago and H. Magalhães and A. Neves and P. Rocha, "On-line multiple model switching control implementation: A case study", in Proc. $10^{\text {th }}$ Mediterranean Conference on Control and Automation (MED'02), Lisbon, Portugal, 2002.

[15] B. Weatherley and S. Williams and E. Neill, Pharmacokinetics, Pharmacodynamics and Dose-Response Relationships of Atracurium Administered i. v., Br. J. Anaesth., vol. 55, 1983, pp 39s-45s.

[16] K. Åström and T. Hägglund, Automatic tuning of PID controllers, Instrument Society of America, 1988.

[17] The Datex-Ohmeda Website: http://www.datex-ohmeda.com/clinical/cw_prev_01_article5.htm

[18] The BIBraun Website:

http://www.bbraunusa.com/products/infusion/perfusor_c1.html 